\title{
1. CiUdad EdUCADORA Y NUEVOS ESPACIOS DE EDUCACIÓN PARA LA SALUD EN LAS PERSONAS MAYORES
}

M. ${ }^{a}$ Rosario Limón Mendizábal y

Juan A. Crespo Carbonero

Universidad Complutense de Madrid

\section{HACIA UN CONCEPTO DE CIUDAD EDUCADORA}

En el prólogo de la obra «Instituciones educativas para adultos en España" señala el profesor Jaume Sarramona que: "todavía estamos muy lejos de la pretendida "ciudad educativa" y apenas conseguimos elaborar algunas "redes educativas" para dar respuesta al principio universalmente aceptado de que la educación es una tarea permanente, que ocupa toda la vida del hombre. Pero de lo que no cabe duda es que la dinámica provocada por la diversidad de iniciativas culturales y formativas ha roto la vieja creencia de que educación es igual a escolarización" (1986:7). Sin duda, la educación se ha orientado a la niñez, a la juventud y a la edad adulta desde una perspectiva economicista. Y la etapa de la Tercera Edad, la más avanzada de la evolución y desarrollo personal, ha sido, hasta comienzos de la década de los años ochenta, la gran olvidada de la Pedagogía. También un ámbito de la educación relegado ha sido la Educación para la Salud. En el desarrollo de ésta, ha sido fundamental la progresiva consolidación de la Pedagogía Social que ha incorporado la reflexión sobre las prácticas socio-educativas a la construcción de una sociedad democrática que pretende ampliar los marcos de la calidad de vida de los ciudadanos: lo que por una parte no sólo ha abierto las puertas a nuevas instituciones y áreas de educación, sino que también ha posibilitado el surgimiento de nuevas disciplinas que estudian y 
analizan estos nuevos fenómenos educativos (García Martínez et al, 2000:8).

Hoy las disciplinas: Educación para la salud, Pedagogía de la vida adulta y de la vejez o Gerontología Educativa y social (Colom y Orte, 2001; Orduña y Naval, 2001), están en "alza» y van siendo, poco a poco, incorporadas en los planes de estudio de las distintas Facultades de Educación, para la formación de los pedagogos y educadores sociales e, incluso, en la formación de maestros. Queremos señalar la aportación de la profesora Rogelia Perea Quesada, Profesora Titular de Pedagogía Social y Educación para la Salud de la UNED, que puso en marcha en el año 1994 un Curso de Especialista Universitario en Educación para Salud que continúa en la actualidad y que ha permitido la formación de muchos médicos, pedagogos, psicólogos, trabajadores sociales y maestros interesados y preocupados por la formación en esta materia.

Tres hechos clave en el desarrollo de estas disciplinas son: la Constitución Española (1978), que ha impulsado el desarrollo de políticas de Bienestar Social; la Asamblea Mundial del Envejecimiento, celebrada en Viena (1982), donde se reconoce la dimensión educativa en la vejez y la importancia del desarrollo de la educación para la salud con el objetivo de obtener una mejor calidad de vida; y el tercer hecho ha sido una de las notas distintivas de finales del segundo milenio: "el cambio". Señala la profesora Labrador Herraiz (1998:209) que «nuestro mundo cambia y cambia vertiginosamente $y$ nosotros, si somos conscientes, debemos aprender a cambiar con él. Cualquier otra actitud nos situaría en una dimensión regresiva imposible de mantener a corto plazo". En este mismo sentido recogemos una reflexión apuntada en el documento elaborado en 1996 por la Unión Europea para la celebración del Año Europeo de la Educación y de la Formación permanentes:

"el objetivo de este Año es sensibilizar a los europeos de la necesidad de formarse durante toda la vida... La responsabilización de la persona, en cuanto a su educación y formación, es aún más necesaria, ya que la propia división tradicional de la sociedad en un periodo de educación, un periodo de trabajo y un periodo de jubilación, ha quedado obsoleta, frente a una perspectiva de renovación constante de las competencias". La educación es una necesidad en todas las edades de la vida y también un derecho.

La expresión ciudad educadora se populariza a principios de los años setenta. Fueron Edgar Faure y otros autores quienes elaboraron para la Unesco el Informe "Aprender a ser» y se manifiesta en él la complejidad y el carácter permanente del hecho educativo, su continuidad fuera de la institución escolar y la posibilidad de utilizar con fines educativos recursos y medios del entorno. También esto es puesto de manifiesto en el Informe coordinado por Jacques Delors "La educación encierra un tesoro" (1996). Queremos subrayar la contribución al estudio de este concepto (ciudad educadora) de los profesores Ortega Esteban (1990, 1998), Trilla Bernet (1990, 1993) y García Garrido (2000). 


\subsection{DIEZ IDEAS ACERCA DEL CONCEPTO DE CIUDAD EDUCADORA:}

1. El concepto de ciudad educadora concibe al medio urbano, a la vez, como entorno, agente y contenido de la educación:

- Entorno: contexto de instituciones y acontecimientos educativos.

- Agente: un vehículo, un instrumento, un emisor de educación.

- Contenido: la ciudad constituye, en sí misma, un objeto de conocimiento.

2. El concepto de ciudad educadora connota muy adecuadamente la complejidad del fenómeno educativo.

- En la ciudad los roles educativos son muchos e intercambiables, las fuentes de conocimiento son incontables (escuelas, museos, bibliotecas, escaparates...(Colom et al, 1987).

3. El concepto de ciudad educadora refiere un medio que produce relaciones y efectos educativos premeditados y también azarosos.

4. Acoge e interrelaciona procesos educativos formales, no formales e informales.

- La ciudad educadora es un entramado de instituciones y lugares educativos.

5, El concepto de ciudad educadora afirma la condición sistémica de lo educativo y demanda planteamientos integradores.

6. El concepto de ciudad educadora afirma el carácter abierto, dinámico y evolutivo de la misma.

- Abierto: interacciona, se nutre, aporta y se prolonga en otros sistemas: medio rural, otras ciudades...

- Dinámico: tiene capacidad de modificar su funcionamiento.

- Evolutivo: tiene capacidad para modificar su estructura para ir optimizando su dimensión educativa.

7. El concepto de ciudad educadora pretende abarcar todas las dimensiones de la educación integral (formación social, intelectual, física, estética y moral, la formación para el trabajo y para el ocio, adquisición de destrezas, de hábitos saludables de vida, de actividades y valores). 
- La ciudad contiene referentes y modelos y se erige en agente para su transmisión.

8. El concepto de ciudad educadora se reconoce en el concepto de educación permanente.

- La ciudad no establece límites temporales a la formación ni discrimina edades para el aprendizaje. Refiere a la vez realidades y utopías.

- El concepto de ciudad educadora advierte que la ciudad no es igualmente educativa para toda la ciudadanía. Igualdad de oportunidades y derecho a la diferencia (Fernández Solis, 1994).

\subsection{LA PEDAGOGÍA URBANA: MARCO DE REFERENCIA DE LA CIUDAD EDUCADORA.}

- La Pedagogía urbana surge de la síntesis entre Educación y Ciudad.

- La Pedagogía urbana establece un marco de referencia en donde las diversas prácticas de las ciudades educadoras se explicitan y se manifiestan conceptualmente tanto a nivel teórico, como en su perspectiva practicista e innovadora.

- La Pedagogía urbana tiene como tareas básicas:

1. Conceptualizar los nuevos espacios educativos.

2. Definir sus áreas de intervención y los puntos de apoyo que las diversas ciencias sociales aportan.

- La Pedagogía urbana aborda la solución de los problemas planteados en la ciudad y la solución de problemas educativos desde la escuela y la ciudad, atendiendo tanto a las necesidades urbanas (desigualdades, drogodependencia juvenil, asociacionismo...) como las necesidades pedagógicas (medios didácticos y fuentes de conocimientos).

\subsection{APORTACIONES DE LA PEDAGOGÍA URBANA}

Se resumen en los siguientes puntos:

1. Ampliación del campo de acción de la Pedagogía.

- La ciudad como espacio-objeto de atención y acción socio-pedagógica. 
2. Aportaciones a nivel ideológico educativo.

- Profundización en los valores sociales y democráticos.

3. Aportaciones de carácter social.

- A través de programas de educación compensatoria, sanitario, preventivos, asistenciales.

4. Aportaciones de carácter didáctico.

- Enseñanza de los contenidos culturales que aporta la ciudad.

5. Aportaciones sobre la personalidad de los sujetos.

- Formación humana y profundización en los valores y en la axiología cívica.

6. Aportaciones de carácter antropológico y cultural.

- Estudios e investigaciones sobre formas urbanas y de vida de las ciudades.

7. Aportaciones de carácter educativo-institucional.

- Acciones orientadas a las mejoras pedagógicas de las escuelas y de otras instituciones consideradas a través de la educación permanente como educadoras de los individuos a lo largo de la vida.

8. Aportaciones pedagógico-curriculares.

- Integración de lo urbano en la escuela.

9. Aportación de servicios educativos y culturales diversos.

10. Aportación de profesionales expertos en educación social. Educadores y pedagogos sociales, etc.

\section{ESPACIOS EDUCATIVOS PARA MAYORES}

La rápida evolución de las características y dimensiones de la población mayor de 65 años ha dado lugar, entre otros muchos fenómenos, a un importante desarrollo de servicios y programas destinados a hacer frente a un conjunto heterogéneo de situaciones de necesidad que experimentan algunas de estas personas. 
La amplitud y diversidad de fórmulas que se han ido desarrollando van desde la apertura de espacios y mecanismos de participación social de las personas y de los grupos, hasta la oferta de programas culturales y de ocio mediante los que ocupan parte del tiempo libre tras la jubilación, pasando por un gran elenco de programas de atención sociosanitaria.

La historia de la implantación de un sistema público de servicios sociales es bastante reciente en España. El marco constitucional (1978), y muy especialmente los artículos 43 y 50 de este texto, sitúan el punto de partida del desarrollo de las políticas de atención a las personas mayores y de educación para la salud.

"Los poderes públicos garantizarán, mediante pensiones adecuadas y periódicamente actualizadas, la suficiencia económica a los ciudadanos durante la tercera edad. Asimismo, y con independencia de las obligaciones familiares, promoverán su bienestar mediante un sistema de servicios sociales que atenderá sus problemas especificos de salud, vivienda, cultura y ocio". (art. 50).

A partir de este marco referencial, en el que el Estado asume el papel de garante del bienestar de sus ciudadanos mayores, las 17 Comunidades Autónomas redactan sus respectivas leyes de Servicios Sociales, desarrollando legislativa y conceptualmente un amplio sistema de atención a las personas mayores.

Las décadas de los años ochenta y noventa han supuesto la implantación definitiva del actual modelo de servicios. Se ha producido un considerable incremento de los recursos sociales más tradicionales (hogares y clubes sociales de jubilados, residencias) y, al tiempo, se ha ido poniendo en marcha una amplia gama de servicios de carácter más innovador (Centros de día, teleasistencia, sistemas alternativos de alojamiento, Universidades para Mayores, Aulas de la Tercera Edad, Cursos de preparación a la jubilación, Centros de Mayores, Centros Cívicos, Centros Sanitarios, Programa de vacaciones, Programa de Balnearios, Voluntariado (formación de voluntarios), Medios de comunicación: programas de radio y televisión y revistas especializadas en mayores, Universidades Populares, Escuelas de adultos, etc. El desarrollo de unos y otros está suponiendo cambios cualitativos importantes en los principios ideológicos y filosóficos que rigen las políticas sociales de atención a las personas mayores (Rodríguez y Sancho 1995). Así, por ejemplo, mientras que a principios de la década de los años 80 el objetivo prioritario de los servicios públicos se centraba en la construcción de residencias dirigidas fundamentalmente a personas con un buen nivel de autonomía funcional, pero con importantes problemas socioeconómicos, en la actualidad el diseño y la planificación de los servicios de atención a las personas mayores suele priorizar aquéllos que favorecen la permanencia en su entorno habitual, con especial énfasis en la atención domiciliaria. Según esta concepción, las residencias y otros servicios institucionales quedan 
reservados para aquellas personas, generalmente mayores de 80 años, con problemas de dependencia funcional o cognitiva tan importantes como para que les imposibiliten seguir viviendo en su medio habitual con niveles mínimos de bienestar.

El desarrollo de los servicios sociales en los últimos años ha evidenciado la necesidad de diseñar planes globales de intervención que respondan a los diversos estados de necesidad de las personas mayores y que, en la medida de lo posible, ofrezcan recursos acordes a estas situaciones. En este sentido, entre todas las programaciones realizadas, merece la pena destacar, como referente de la política de Estado para este grupo de población, el Plan Gerontológico (INSERSO, 1993), marco de actuación para la década de los años 90, y que está basado en el Plan de Viene (1982). Se trata de un diseño de intervención con un enfoque de atención integral, que se sistematiza en torno a cinco grandes áreas: Pensiones, Salud y Asistencia Sanitaria, Servicios Sociales, Cultura y Ocio y Participación. En cada una de ellas se establecen grandes líneas de actuación, objetivos y medidas para la cumplimentación de los mismos, así como los Organismos responsables para llevarlas a efecto. A lo largo del texto de dicho Plan se va exponiendo un conjunto muy amplio de servicios y programas para las personas mayores, muy especialmente los que se circunscriben a los ámbitos social y sanitario, y también los relacionados con el acceso a los bienes culturales, al ocio y a la participación de este grupo de población en la vida social.

Señala M. ${ }^{\text {a }}$ Teresa Sancho (Directora de Programas del Plan Gerontológico, IMSERSO) que quizá deba valorarse el Plan Gerontológico más que por el propio desarrollo de servicios que él mismo ha impulsado, por haber contribuido de manera decisiva a un cambio conceptual en la consideración de las necesidades de las personas mayores y en la interpretación del fenómeno del envejecimiento de la población de las sociedades modernas.

Describimos a continuación algunos de los Espacios para mayores, donde se favorecen las relaciones de convivencia y desarrollo integral de las personas mayores. Son también espacios de aprendizaje, donde se ofrecen programas de prevención, permitiendo el conocimiento de hábitos saludables para favorecer el desarrollo de la autonomía personal.

\subsection{LOS HOGARES Y CLUBES}

Son centros de reunión que promueven la convivencia de las personas mayores y la mejora de sus condiciones de vida a través de diversos servicios (INSERSO, 1995). 


\section{Cuadro1. Servicios que suelen of recer los hogares.}

- Información, orientación y asesoría

- Biblioteca

- Cafetería y servicio de comidas

- Peluquería

- Podología

- Actividades lúdicas y recreativas (excursiones, bailes, fiestas, cursos y talleres, etc.)

- Actividades sociales y formativas

- Animación y cooperación social (voluntariado, relaciones intergeneracionales, etc)

Además de servicios como los reseñados, en algunos hogares o clubes, sobre todo los dependientes de alguna de las Administraciones Públicas, también se suministran otros servicios asistenciales de índole sanitaria y/o de atención para personas con problemas de dependencia: prevención sanitaria, chequeos de salud, ayuda a domicilio, distribución de comida y lavandería a domicilio, etc.

Si bien en algún momento la valoración de los hogares no ha sido muy positiva, por sus indudables rasgos de marginalidad, una evaluación más profunda y a largo plazo de los mismos muestra el importante papel que estos centros han desempeñado, y aún hoy continúan cumpliendo. De hecho, han contribuido de forma significativa a reforzar y crear redes de apoyo social entre las personas mayores con todos los beneficios que esto conlleva, a implantar hábitos de vida más saludables y, en definitiva, a mejorar la calidad de vida de este grupo de población.

Desde algunos lugares se comienza a considerar estos centros como capaces de convertirse en el eje de un conjunto polivalente de programas y servicios de manera que, sin renunciar a su primigenio carácter de lugar de encuentro entre las personas mayores del lugar, sirvan como centros dispensadores de servicios comunitarios.

\subsection{CENTROS DE DÍA}

Se define este recurso como un "servicio socio-sanitario y de apoyo familiar que ofrece durante el día atención a las necesidades personales básicas, terapéuticas y socio-culturales de personas mayores afectadas por diferentes grados de dependencia, promoviendo su autonomía y la permanencia en su entorno habitual" (Sancho, 1996). 
El objetivo general del centro de día es "favorecer unas condiciones de vida dignas entre las personas mayores dependientes y sus familiares, facilitando la continuidad en sus modos de vida y el logro de un mayor nivel de autonomían.

Es éste un servicio de los de más reciente implantación en España, por lo que el número de centros puestos en marcha desde la iniciativa pública y privada es poco significativo y es necesario para los próximos años disponer de un mayor número de plazas.

\section{A) ATENCIÓN SOCIAL:}

- Detección, acogimiento, orientación y seguimiento de casos, coordinación del plan de intervención establecido, relación con la familia, etc.

- Establecimiento del plan de transporte

B) ATENCIÓN A LA SALUd PSICO-FISICA:

- Rehabilitación y control psico-físico

- Actividades psicoterapéuticas

- Terapia ocupacional

- Higiene personal

- Dietética

\section{C) SOCIALIZACIÓN Y PARTICIPACIÓN:}

- Entrenamiento en habilidades sociales

- Relaciones personales

- Actividades de ocio y cultura

- Actividades productivas

\subsection{CENTROS RESIDENCIALES}

Otro espacio de los mayores son las residencias de ancianos. Durante mucho tiempo, fueron consideradas la única solución a los problemas de aislamiento y de pérdida de autonomía funcional relacionados con la vejez. Posteriormente, cuando empezaron a manifestarse los problemas derivados del ingreso en estos centros, las políticas sociales comenzaron a privilegiar el mantenimiento de los ancianos en su medio doméstico originario durante el mayor tiempo posible. 
En los últimos años, además, han aparecido nuevas modalidades de alojamiento para gente mayor (viviendas tuteladas, apartamentos con servicios, acogimiento de un mayor por una familia, etc.).

El Instituto Nacional de Servicios Sociales (1993) define las residencias como «centros que ofrecen atención integral y vivienda permanente a personas mayores de 60 años que, por su problemática familiar, social y/o económica, no pueden ser atendidas en sus propios domicilios y necesitan de estos servicios».

\section{Cuadro 3. Programas de Intervención en Residencias}

\section{SANITARIOS}

- Atención médica

- Fisioterapia

- Terapia ocupacional

\section{PSICO-SOCIO-EDUCATIVOS}

- Atención psicológica

- Intervención con familias

- Animación socio-cultural

\section{PARA CASOS DE DETERIORO COGNITIVO}

- Orientación a la realidad

- Entrenamiento de habilidades básicas

\section{CUIDADOS PALIATIVOS}

Poco a poco las residencias de ancianos en España se van alejando, aunque quizás no todas, de la imagen de aquellos asilos que eran espacios cerrados, ubicados fuera de las ciudades y donde el anciano lo único que esperaba era la muerte. Sin duda en este cambio de concepto ha tenido mucho que ver el desarrollo del Plan Gerontológico Nacional (1993), en donde se plantea que la residencia, como centro gerontológico, sea un espacio abierto a la comunidad, a la participación, a las relaciones humanas, a la solidaridad, a la cultura, que incorpore a la vida del centro agentes externos (ya sean familiares, amigos de los residentes u organizaciones de la iniciativa social y ciudada- 
na), y que el protagonista principal sea la propia persona mayor. Y señala como medida introducir progresivamente en los centros gerontológicos la figura del animador sociocultural (1993:147).

En el Manual de orientación "Residencias para personas mayores», Pilar Rodríguez, coordinadora de esta obra, define la residencia como "Centro gerontológico abierto, de desarrollo personal y atención socio-sanitaria interprofesional, en el que viven temporal o permanentemente personas mayores con algún grado de dependencia» (1995:39).

La clasificación tradicional que distinguía residencias para personas mayores "válidas» o "asistidas" está siendo sustituida por una única tipología acorde con su definición, es decir, centros para personas con problemas de dependencia. En consecuencia, se está produciendo un rápido proceso de reconversión de las plazas para personas válidas, impuesta por la evidencia de una progresiva pérdida de capacidad funcional de las personas que las ocupan.

\subsection{ESTANCIAS TEMPORALES EN RESIDENCIAS}

Es éste otro servicio de carácter innovador en nuestro país y de reciente implantación especialmente desde las Administraciones Públicas.

Este programa posibilita la estancia en un centro residencial, durante un período generalmente no superior a 60 días, a aquellas personas mayores de 65 años que se encuentran, bien ellas mismas o las familias con quienes conviven, en algún estado eventual de necesidad, susceptible de solución o mejora mediante el acceso a este recurso (INSERSO, 1994).

Es un servicio que también sirve de apoyo familiar, al igual que el centro de día, al cumplir una importante función de alivio para los cuidadores, favoreciendo así la permanencia de la persona mayor en el entorno familiar y, en consecuencia, evitando la institucionalización definitiva.

Los programas de estancia temporal en una institución ofrecen los mismos servicios de los que disfrutan las personas que residen permanentemente en éstas. No obstante, debe elaborarse siempre un plan de intervención personalizado para cada caso en el que se prevean, además de los consiguientes objetivos de carácter terapéutico-rehabilitador, las medidas que favorezcan una adecuada adaptación al centro y posterior reinserción familiar.

\subsection{SISTEMAS ALTERNATIVOS DE ALOJAMIENTO}

Bajo esta denominación se agrupa una gama de alojamientos para personas mayores que, por problemas de vivienda, soledad o pérdida de autono- 
mía, se ven obligadas a abandonar su domicilio y optan por las soluciones más parecidas a su modo de vida habitual (Sancho, 1994).

\section{Cuadro 4. Características de los alojamientos alternativos}

- Ubicados en el entorno habitual

- Convivencia en grupos pequeños

- Organizados en torno a algún sistema de tutela

- Diseño hogareño, no institucional

- Autofinanciación en muchos casos por sus inquilinos

La oferta que existe es bastante diversa y se concreta en modalidades como las siguientes:

a) Apartamentos individuales o bipersonales, con una gama más o menos amplia de servicios que se ofrecen en el mismo edificio o en algún centro gerontológico cercano: lavandería, restaurante, comidas a domicilio, asistencia doméstica, servicios médicos y de enfermería, actividades de ocio y animación, etc.

La intensidad y modalidades de estos servicios complementarios varían en función del grado de dependencia de sus ocupantes, procurando adaptarse a sus diferentes estados de necesidad.

Estos apartamentos, que oscilan entre los 30 y $50 \mathrm{~m}^{2}$, suelen disponer de algún tipo de teleasistencia o simplemente de una alarma conectada a la conserjería del edificio, lo que proporciona seguridad a sus inquilinos.

b) Viviendas tuteladas, ocupadas por grupos que oscilan entre 4 y 10 personas.

c) Viviendas comunales, que habitualmente son autogestionadas por las propias personas mayores que las ocupan.

d) Minirresidencias, de hasta 20 personas, desarrolladas sobre todo en el medio rural.

e) Acogimiento, en diversas fórmulas, como acogida familiar, de convivencia intergeneracional, etc.

Básicamente, la tipología de alojamientos enumerada podría sintetizarse en torno a dos grupos: el formado por alternativas convivenciales y el que opta por alternativas individuales. 
El tipo de tutela que se ejerce en los diversos tipos de alojamiento varía mucho, siendo determinante el grado de dependencia de los miembros del grupo, así como el modo de convivencia que se practique: relaciones informales similares a las que se establecen en una pensión, ambiente de camaradería y apoyo mutuo, vida cuasi-familiar, etc.

\subsection{SERVICIOS DE AYUDA A DOMICILIO}

El servicio de ayuda a domicilio se presta en España, fundamentalmente, por iniciativa de las Administraciones Públicas y, sobre todo, por las corporaciones locales. La gestión y prestación de este programa suele correr a cargo de organizaciones de carácter no lucrativo, así como por empresas mercantiles o cooperativas de servicios, con quienes los ayuntamientos suscriben los correspondientes conciertos de servicios.

Ha sido definido como un "programa individualizado, de carácter preventivo y rehabilitador, en el que se articulan un conjunto de servicios y técnicas de intervención profesionales, consistentes en atención personal, doméstica, de apoyo psicosocial y familiar y relaciones con el entorno, prestados en el domicilio de una persona mayor dependiente en algún grado» (Rodríguez, 1997).

\section{Cuadro 5. Objetivos del Servicio de Ayuda a Domicilio (SAD)}

- Facilitar la autonomía de sus usuarios

- Conseguir cambios conductuales que mejoren la calidad de vida

- Fomentar hábitos saludables

- Aumentar la seguridad personal

- Potenciar las relaciones sociales

- Lograr la permanencia en su medio habitual de vida en condiciones de dignidad

- Evitar la institucionalización innecesaria

Los servicios que se ofrecen a través del SAD son muy diversificados. Van desde todo el conjunto que tiene que ver con la realización de tareas domésticas a la atención personal y ayuda en las actividades de la vida diaria (AVD), pasando por el cuidado y apoyo a las personas con graves dependencias (movilizaciones, manejo de sondas, etc.), sin olvidar las actividades que facilitan la integración social. 


\section{Cuadro 6. Servicios que sucle ofrecer el SAD}

- Limpieza de la casa

- Lavado y planchado de la ropa

- Compra y preparación de comidas

- Aseo personal

- Movilizaciones

- Vestirse/desvestirse

- Peluquería

- Cuidados sanitarios elementales

También se prestan otros servicios complementarios, como son: acompañamiento, realización de gestiones, actividades de ocio, etc. Los servicios de teleasistencia, así como los de comidas a domicilio y lavandería se incluyen en ocasiones por algunas entidades dentro de estos servicios complementarios.

Las asociaciones de personas mayores y otras entidades de voluntariado están desarrollando de forma considerable los servicios complementarios. La coordinación entre la provisión de servicios de atención domiciliaria de carácter social y sanitario se configura como objetivo prioritario de intervención.

\subsection{PROGRAMAS DE VACACIONES}

Desde mediados de los años 80 se promueven desde las diferentes administraciones públicas programas subvencionados de vacaciones. En un principio los viajes se realizaban en Navidad e iban dirigidos a personas que vivían solas. Entre todas las actuales ofertas, destaca la que se realiza por el IMSERSO, cuyo programa, iniciado en la temporada $1985-86$ con 16.000 personas, ha desembocado en una iniciativa de tanta importancia como que en el año 2001 han podido disfrutarlo 400.000 personas. De ellas, casi 17.000 han correspondido a españoles, hoy jubilados, que emigraron durante su etapa de actividad laboral a algún país de Europa, Iberoamérica, norte de África y Australia. Básicamente consiste en ofrecer, a precios muy reducidos, plazas hoteleras en temporada baja (de octubre a junio) en determinadas zonas turísticas, sobre todo de la costa mediterránea y las islas Baleares y Canarias y también Portugal.

Dos objetivos fundamentales del Programa son:

1. Contribuir a la mejora del bienestar de las personas mayores, mediante el disfrute de la actividad turística. 
2. Favorecer la creación y mantenimiento de empleo en el sector de la hostelería de las costas españolas, rompiendo así su carácter estacional.

El perfil más típico de los usuarios de este programa responde al de una persona de edad no muy avanzada (menos de 75 años) que reside en núcleos urbanos, vive con su pareja en su propio domicilio y tiene un nivel cultural similar al de la media de este grupo de población. Sus principales motivaciones para realizar estos viajes son el deseo de conocer nuevos lugares y el de pasar unos días de descanso y distracción.

Existe otra modalidad de este programa, consistente en la realización de Circuitos culturales por el interior y norte de España. Este tipo de viajes responde a las nuevas demandas de un sector de población que empieza a interesarse por una oferta con mayor contenido cultural y que posibilita en muchos casos el reencuentro con sus raíces y la ampliación de sus conocimientos.

\section{Cuadro 7. Servicios que incluye el Programa de Vacaciones del IMSERSO}

- Desplazamientos de ida y vuelta desde el lugar de origen al punto de destino

- Estancia en hoteles de hasta cuatro estrellas de 15 días de duración ( 6 días en el caso de los circuitos culturales) en régimen de pensión completa.

- Actividades de ocio y tiempo libre

- Atención sanitaria

- Póliza de seguros

\subsection{PROGRAMA DE TERMALISMO}

Lo mismo que en el programa de vacaciones, también con respecto al de termalismo se realizan ofertas a las personas mayores desde diferentes instituciones. Pero también en este caso destaca por su importancia el programa promovido y desarrollado por el Ministerio de Trabajo y Asuntos Sociales, a través del IMSERSO. Éste organiza desde hace años estancias de quince días de duración en establecimientos termales especializados para las personas mayores que por prescripción facultativa las necesiten. El programa comenzó en el año 1989 con una participación de 12.848 personas mayores. En el año 2001 se han ofrecido 85.000 plazas.

Es una iniciativa que ha suscitado gran interés entre las personas mayores, que pueden disfrutar del programa a precio reducido. El desarrollo del 
programa de termalismo ha supuesto también un relanzamiento considerable de este sector, que conjuga el ocio y el tratamiento terapéutico. Tal ha sido el impacto, que se ha incrementado considerablemente la demanda de los balnearios también entre otros grupos de edad. Su eficacia en relación con la mejora de la calidad de vida ha sido constatada mediante estudios de evaluación del programa (INSERSO, 1997).

\subsection{UNIVERSIDADES POPULARES}

Tiene ya bastante tradición la oferta que se realiza a las personas mayores desde las UU.PP. Éstas son centros de educación de adultos y de animación sociocultural, normalmente financiadas por una Administración Pública (CC.LL., CC.AA., etc)

Dentro del Área socioeducativa de las UU.PP. se pueden encontrar ofertas en la planificación muy interesantes para las personas mayores.

Cuadro 8. Programas que suelen ofrecerse en las Universidades Populaires

- Educación básica: alfabetización, neolectura, preparación para graduados escolares, etc.

- Cursos y talleres para ampliar conocimientos culturales: historia, arte, literatura, etc.

- Cursos y talleres relacionados con el incremento de la calidad de vida: ecología, alimentación, salud, etc.

- Talleres de expresión y creación plástica: teatro, danza, música, artesanía, etc.

\subsection{AULAS DE PERSONAS MAYORES}

La iniciativa de este tipo de oferta fue en su inicio de las Administraciones Públicas (sobre todo de las Comunidades Autónomas). Sin embargo, en la actualidad casi siempre la gestión y la responsabilidad en la programación de las aulas corresponden a las propias asociaciones de personas mayores. Los programas que se ofrecen son muy diversificados, pero casi siempre se inscriben en alguno de los ámbitos siguientes: cultural, ocupacional, de desarrollo físico-psíquico, de acción social y convivencia. La programación suele ser del tipo de la señalada en el apartado anterior (Universidades Populares). 


\subsection{PROGRAMAS UNIVERSITARIOS PARA PERSONAS MAYORES}

Hace sólo unos años era impensable que la Universidad brindara la posibilidad de participar en la enseñanza activa a las personas mayores, como también que la educación estuviera presente y fuese necesaria en esta etapa de la vida. Hoy es una realidad para muchos mayores este nuevo espacio socio-educativo.

Con estos Programas se pretende ofrecer a los mayores la participación en el mundo universitario, de modo que puedan tener la satisfacción que proporciona el saber y la vinculación humana con personas de intereses similares en un medio tan estimulante como es la Universidad.

Los Programas Universitarios de Mayores se están implantando progresivamente en todas las Universidades del mundo desarrollado. En España hay actualmente más de cuarenta Universidades que están desarrollando estos Programas; es interesante la Guía de Programas Universitarios de Personas Mayores editada por el Ministerio de Trabajo y Asuntos Sociales (1999).

Velázquez Clavijo y Cristina Fernández señalan en esta Guía (1999:15), que son varias las razones que han impulsado la implantación y desarrollo de estos Programas: "el creciente incremento de la esperanza de vida; el compromiso de las autoridades académicas universitarias de apostar por un nuevo sistema de formación permanente; el interés social suscitado por este sector de la población, debido al mucho tiempo de que disponen como consecuencia de su jubilación; el desarrollo cada vez mayor de las investigaciones y publicaciones en el ámbito de las personas mayores y, por último, la coherente y solidaria filosofía de abrir la Universidad a la sociedad con la intención de dar respuesta a uno de los sectores más desprotegidos de la población: las personas mayores".

Estas y otras razones, justifican, desde nuestro punto de vista, el actual florecimiento de los Programas Universitarios de Mayores en España, como respuesta a la creciente demanda de este sector de población.

M. ${ }^{a}$ Adoración Holgado, directora de la Universidad de la Experiencia (Universidad Pontificia de Salamanca) apunta que estos Programas, al abrir las aulas de la Universidad a hombres y mujeres sin más requisitos que la edad, generalmente a partir de los 55 , pueden justificarse tanto desde la perspectiva individual al permitir y facilitar que algunas personas puedan elegir, entre otras propuestas, una oferta cultural universitaria, como desde la perspectiva social intentando saldar una deuda histórica que la Universidad como Institución tiene con muchas personas para las que sus aulas estuvieron vetadas durante años (1999:19). 
Consideramos de interés exponer en el cuadro núm. 9 los niveles educativos de la Tercera Edad española. Como se observará, los niveles educativos de este sector de población son notablemente bajos, sin duda debido, entre otras causas biográficas, al hecho de que durante su niñez y juventud la enseñanza era privilegio de una minoría, puesto que la educación formal en aquellos tiempos no era exigida. También debemos añadir que la precariedad cultural de muchos de nuestros mayores es debida a que desde sus años jóvenes han sufrido la ruptura, la interrupción o abandono en el proceso educativo para incorporarse precozmente al trabajo.

Sólo 156.657 personas mayores han realizado estudios universitarios, lo que nos dice a las claras la tremenda marginación y discriminación de esta generación respecto a la educación.

\section{Cuadro núm. 9. Nivel de instrucción de la Tercera edad}

\begin{tabular}{|lrrr|}
\hline $\begin{array}{l}\text { Población de más } \\
\text { de 65 años }\end{array}$ & \multicolumn{1}{c|}{ Total } & Varones & Mujeres \\
& & & \\
ANALFABETOS & 567.073 & 124.036 & 443.037 \\
SIN ESTUDIOS & 2.515 .758 & 1.032 .451 & 1.483 .306 \\
PRIMER GRADO & 1.701 .803 & 730.953 & 970.849 \\
SEGUNDO GRADO & 359.324 & 189.212 & 170.112 \\
TERCERA GRADO & 156.657 & 104.844 & 51.813 \\
NO CONSTA & 51.737 & & \\
$\quad$ SUMA & $\mathbf{5 . 3 5 2 . 3 5 2}$ & & \\
\hline
\end{tabular}

Fuente: Censo de población de 1991, I.N.E., Madrid, 1992.

SIN ESTUDIOS:

PRIMER GRADO:

SEGUNDO GRADO:
Incluye las personas que saben leer y escribir pero no han asistido a la escuela o han asistido menos de cinco años

Estudios primarios/Cinco cursos aprobados de E.G.B. o equivalente.

Primer ciclo: Bachiller elemental (General, Laboral o Técnico) - Graduado Escolar - E.G.B. completa o equivalente. Segundo ciclo: Formación Profesional Primer grado, Oficialía industrial Formación Profesional Segundo grado, Maestría industrial. Bachiller Superior, B.U.P. 
Otras titulaciones medias: Artes aplicadas y oficios artísticos, cerámica, conservatorio de música y escuelas de arte dramático y danza, escuelas oficiales de idiomas y cualquier otro estudio que exija para cursarlo el título de graduado escolar o equivalente.

TERCER GRADO:

Arquitecto e Ingeniero Técnico - Diplomado de Escuelas Universitarias y de Facultades - Arquitecto e Ingeniero superior - Licenciado universitario - Doctorado - Estudio de Postgrado - Otras titulaciones superiores.

\section{Ojetivos de los programas universitarios de mayores}

Entre los Objetivos de los Programas Universitarios de mayores recogemos los de las Universidades Autónoma de Madrid, de las Islas Baleares, Pontificia de Salamanca y Sevilla $(1999,53: 56)$.

U. Autónoma de Madrid «Vivir con vitalidad»

U. de Islas Baleares "Universidad Oberta a Majors»

U. Pontificia de Salamanca

"Universidad de la

Experiencia»
- Transmitir conocimientos sobre cómo envejecer saludable y competentemente

- Potenciar estilos de vida saludables

- Modificar las imágenes negativas existentes en la vejez y el envejecimiento.

- Promover la participación en actividades culturales, físicas y mentales

- Abrir la Universidad con una oferta específica para personas mayores

- Fomentar la participación de los mayores en la sociedad actual

- Potenciar otra percepción e imagen de las personas mayores

- Divulgar los diferentes ámbitos de conocimientos y experiencias entre las personas mayores

- Posibilitar el intercambio de conocimientos y experiencias entre las personas mayores

- Hacer posible las relaciones intergeneracionales

- Intercambiar conocimientos y experiencias entre personas mayores

- Fomentar la participación de los mayores en la sociedad actual 
U. de Sevilla

"Aula de la Experiencia"
- Profundizar y/o divulgar diferentes campos de la cultura

- Facilitar un espacio para el debate científico cultural

- Ofrecer un marco para las relaciones intergeneracionales

- Proporcionar, de forma activa, el acceso de las personas a los bienes culturales para la mejora de la calidad de vida y el fomento del empleo creativo del ocio.

- Fomentar la participación de las personas mayores como dinamizadores en su contexto socio-cultural

Llegar a la vejez en un estado de bienestar y buena salud requiere esfuerzo individual a lo largo de toda la vida y un entorno en que dicho esfuerzo pueda tener éxito. La responsabilidad de la persona es mantener un estilo de vida saludable, mientras que la responsabilidad de los gobiernos y de otros organismos e instituciones es crear un entorno propicio que permita que la salud y el bienestar avancen hasta la vejez.

Todos estos Espacios y Programas (antes analizados) son espacios de educación para la salud, espacios de aprendizaje y de vida, para compartir información, experiencias y formación, con el fin de potenciar el desarrollo personal en esta nueva etapa de la vida. Entramos en planteamientos de educación permanente.

\section{TEMAS PARA UN «CURSO DE PROMOCIÓN DE LA SALUD Y BIENESTAR EN LOS MAYORES"}

El programa que presentamos con el título "Promoción de la salud y bienestar en los mayores» está diseñado para animar grupos de mayores siguiendo, orientativamente, la metodología propuesta en el libro Grupos de debate para mayores (Limón Mendizábal, M. R. y Crespo Carbonero, J. A., 2001).

Los objetivos, los temas de estudio y reflexión que se ofrecen en los doce temas del curso enunciado pueden ser desarrollados en los distintos espacios dirigidos a las personas mayores. Son los siguientes:

1. Salud y bienestar en la vejez. 2. Prevenir los accidentes caseros en la vejez. 3. Cuidar los cinco sentidos. 4. Los mayores, el estrés y la relajación. 5. El valor de la comunicación y el diálogo en la vejez. 6. Enfermedades que afec$\tan$ a muchos mayores. 7. El alcoholismo entre los mayores. 8. La sabiduría del optimismo realista en la vejez. 9. Los juegos de cartas como espacio de encuentro y desarrollo entre los mayores. 10. Recomendaciones para cuidar tu 
memoria. 11. Mantenerse en forma de mayores con el ejercicio físico. 12. Grupos de ayuda mutua entre los mayores.

A modo de ejemplo, presentamos a continuación tres de ellos.

\subsection{TEMA: SALUD Y BIENESTAR EN LA VEJEZ}

\subsubsection{Objetivos específicos:}

1. Dar la oportunidad de reflexionar y compartir ideas sobre el tema de la salud y el bienestar en la vejez.

2. Ofrecer información práctica que facilite a las personas mayores una buena salud y bienestar durante esta etapa de la vida.

3. Concienciar de la propia responsabilidad de los mayores ante su salud y bienestar.

4. Estimular la creación de hábitos saludables que promuevan una vejez satisfactoria.

5. Reforzar y animar aquellas aptitudes, conductas o reflexiones que fomenten la salud y el bienestar del mayor.

\subsubsection{Texto (para leer, analizar y debatir):}

Estar enfermo representa el peligro más serio y temido para un gran número de mayores. Ante la pregunta ¿qué significa estar sano?, la respuesta más habitual de los mayores es: vivir sin sufrimientos o dificultades que nos impidan ser autónomos en la actividad diaria.

El concepto de salud está ligado al de bienestar y desarrollo personal. La salud es algo más que no estar enfermo, y se debe entender de forma más amplia y positiva, como un proceso en el que las personas colaboran activamente para conseguir el máximo desarrollo de sus capacidades, buscando su realización personal y social.

La salud en la vejez está conectada con el mantenimiento de las capacidades físicas, mentales y sociales.

Las principales características que describen una vejez sana son:

- Estar en contacto con la realidad. Esto es, hacer frente a los problemas de la vida con criterio realista, reconociendo las limitaciones y buscando el desarrollo de las facultades que se poseen.

- Ser uno mismo, esforzándose por mantener la autonomía personal y una convivencia social satisfactoria.

- Tener una vida emocional equilibrada.

- Integrarse en la vida social del propio entorno.

- Poseer confianza en uno mismo, ser activo, creativo y crítico.

- Obtener estímulos positivos en la actividad diaria. 
Dos opiniones equivocadas sobre la salud en la vejez son: primero, identificar vejez con enfermedad, esto es, creer que todos al envejecer enfermamos de una u otra forma; $y$, segundo, pensar que la salud se mantiene principalmente, y casi exclusivamente, a base de medicamentos.

Ninguna de estas dos ideas son correctas. Debemos saber que, en general, los mayores experimentan una vejez sana y que los medicamentos no son la base de la salud, simplemente representan un apoyo al que se debe acudir sólo cuando es necesario y sugerido por profesionales.

Las enfermedades más frecuentes en la vejez son: los accidentes cerebrovasculares, la artritis, la hipertensión arterial, las depresiones, la incontinencia urinaria, las cardiopatías, la bronquitis y asma, la diabetes, las demencias y la sordera.

Algunos de los aspectos que no debemos descuidar para prevenir las enfermedades y desarrollar una vida sana son:

- Estar activo, mantenerse ocupado en actividades de interés.

- Comer y beber adecuadamente.

- Moverse, hacer ejercicio, mantener una posición correcta.

- Dormir, descansar y vestirse adecuadamente.

- Mantener la higiene y el aseo personal.

- Rodearse de un entorno agradable y seguro, que evite los accidentes domésticos.

- Comunicarse y expresar las propias ideas y sentimientos. Sentirse integrados y comprometidos socialmente.

- Evitar el estrés, las drogas, la vida sedentaria, las medicinas innecesarias, el aislamiento, el pesimismo.

- Buscar el mayor grado de autonomía, reconociendo la propia responsabilidad ante la salud.

Para Imanol Ormaza (1995), especialista en enfermería geriátrica, existen dos puntos decisivos, relacionados entre sí, que deben abordarse cuando se revisa el tema de la salud en la vejez; éstos son: la educación para la salud y la promoción de los grupos de apoyo.

La salud es un derecho conectado con el bienestar y la actividad general de todas las personas, de ahí que no deba delegarse sólo en los profesionales sanitarios, y sí tenga que ser considerada como una responsabilidad personal y social, que necesita estimularse por medio de la educación popular y comunitaria.

Desde esta perspectiva, sería altamente saludable que los mayores se comprometiesen en proyectos y actividades intergeneracionales y comunitarias, con el fin de promover la educación para la salud. O sea, que, con actitud educativa crítica, estimularan en las personas de su entorno actitudes positi- 
vas hacia la salud, promoviendo la creación de hábitos y conocimientos al alcance de todos para mantener y mejorar la salud.

La participación comunitaria de los mayores, a través de grupos de autoayuda o de mutuo apoyo, representa un instrumento de gran valor para afianzar el bienestar y la salud en la jubilación. En torno a estos grupos se relacionan personas con intereses o problemas comunes, como la depresión, el alcoholismo, familiares de enfermos, grupos de amigos, vecinos o voluntarios, para recibir colaboración, apoyo emocional, consejos de expertos e información, ayuda personalizada, ánimos para mejorar las condiciones de vida y estímulos para superar las dificultades y continuar autónomos.

\subsubsection{Preguntas para animar y centrar el debate}

1. ¿Cuándo decimos que nos encontramos sanos? ¿qué significa estar sano cuando ya eres un anciano?

2. ¿Es cierto que lo peor que puede pasar a un mayor es caer enfermo?

3. ¿Conoces a algún mayor que haya vivido su vejez con buena salud? ¿Cuáles dirías que son las características que describen una vejez sana?

4. ¿Cuál debe ser el uso correcto de los medicamentos?

5. ¿Cuáles son las enfermedades más habituales en las personas mayores?

6. ¿Merece la pena esforzarse para prevenir una vejez más sana?

7. ¿Qué consejos darías a alguien que se va a jubilar y te pregunta qué se debe hacer para vivir una vejez satisfactoria?

8. ¿Cuáles son los hábitos sanos que los mayores practican en la actualidad y cuáles son los que aún debemos integrar en nuestras costumbres?

9. ¿Qué proyectos o actividades recomendarías a los responsables políticos, para fomentar en tu localidad una vejez saludable y feliz?

10. ¿Sinceramente crees que comprometerse a ayudar a otras personas favorece tu bienestar personal?

\subsection{TEMA: EL VALOR DE LA COMUNICACIÓN Y EL DIÁLOGO EN LA VEJEZ}

\subsubsection{Objetivos específicos:}

1. Ofrecer información sobre el valor saludable de la comunicación amistosa.

2. Dar la oportunidad de reflexionar y compartir ideas sobre el valor del diálogo comunicativo.

3. Concienciar al mayor de su responsabilidad en el cuidado personal de su salud, desarrollo mental y afectivo.

4. Estimular la creación de hábitos saludables y preventivos que promuevan la comunicación en la vejez.

5. Reforzar y animar aquellas actitudes, conductas o reflexiones saludables conectadas con la comunicación. 


\subsubsection{Texto (para leer, analizar y debatir):}

Dicen que el diálogo amistoso, relajado e interesante es una de las experiencias más agradables que nos reserva la vejez. Cuando llegamos a mayores y el desgaste de los años hace que nos cuestionemos si merece la pena seguir viviendo, la comunicación acogedora y gratificante de quienes nos rodean nos anima a seguir viviendo, nos ofrece una oportunidad para dar sentido y valorar positivamente nuestra vida, pese a las dificultades.

Pero no siempre que hablamos con otros logramos comunicarnos satisfactoriamente. Para que el diálogo se convierta en una actividad emancipadora, de convivencia respetuosa y desarrollo, es necesario que actuemos adecuadamente. Esto es, exige promover el diálogo como un encuentro receptivo, en el que los interlocutores comparten experiencias vitales personales, que transmiten algo íntimo de sí mismos, como las ilusiones, los miedos, los logros, las alegrías, los fracasos, las tristezas, los proyectos y las preocupaciones.

El diálogo que acaba siendo comunicación auténtica produce una influencia recíproca entre los interlocutores. Cuando dos personas hablan de algo que les interesa, prestándose atención, profundizando en sus experiencias, buscando una comprensión mutua, algo, por pequeño que sea, cambia en ellos.

Si somos capaces de dar a esta influencia un carácter constructivo y humanizador, encontraremos en el diálogo una terapia asequible para desarrollar nuestras capacidades y prevenir los desequilibrios psíquicos que, en algunas ocasiones, sufrimos al envejecer.

Pese a que en la actualidad todos reconocemos que comunicarse satisfactoriamente es una condición necesaria para disfrutar de la vejez, comprobamos diariamente cómo nuestra sociedad tiende a dificultar la comunicación de los ancianos y a provocar su aislamiento.

La despoblación de las zonas rurales y el crecimiento progresivo de las ciudades promueve que vivamos rodeados de gente por todas partes, pero aislados y con graves problemas de comunicación. No conocemos a la gente que vive en nuestro barrio, y apenas nos limitamos a saludar y cruzar unas palabras de cortesía con nuestros vecinos.

Incluso dentro de la familia los mayores encuentran serias barreras para comunicarse, pasan casi todo el día solos y, cuando llegan a casa los hijos y los nietos, encuentran que los problemas, las prisas y, sobre todo, la televisión, dificultan la comunicación y el diálogo familiar.

Por otra parte, el poco valor que damos a nuestra comunicación y la torpeza de nuestras costumbres pueden reducir nuestro diálogo a simple descalificación y chismorreo sobre otras personas o nos limitamos a transmitir 
información poco relevante sobre programas de televisión o fútbol, sin llegar a profundizar en nuestras experiencias vitales.

Por unas y otras razones, gran cantidad de ancianos suelen «matar su tiempo» solos. Como ha escrito J. José Tamayo: «Cada vez es mayor el número de personas que, por diversos motivos, viven solas, sin nadie que pueda responder a sus preguntas, que tome en serio sus problemas; sin nadie a quien preguntar o a quien dirigirse como un tú personal, que escuche y comprenda, que acoja y estimule; sin nadie con quien reír o llorar y, en definitiva, con quien vivir. Éste es, quizás, el lado más oscuro, desesperanzador y negativo de la existencia humana».

Toda la sociedad y especialmente los mayores deberíamos reflexionar sobre ¿cómo es posible que ocurra esto?, ¿cómo puede suceder que un acto tan simple y accesible como el diálogo constructivo haya llegado a convertirse en algo tan raro y difícil en nuestra sociedad? Si queremos poner medios para disfrutar de una jubilación feliz necesitamos promover el diálogo que fomente la comunicación entre las personas.

Algunos consejos prácticos que pueden facilitarnos la comunicación y el diálogo son los siguientes:

1. Si posee dificultades sensoriales para comunicarse no dude en hacer un esfuerzo por resolverlas. Use audífonos y gradúese periódicamente la vista. Procure mirar de frente a su interlocutor cuando habla y pronuncie con claridad y sin prisas; si no ha oído lo que los otros dicen, solicite que se lo repitan.

2. Utilice todas las formas de comunicación a su alcance para no perder contacto con esos buenos amigos de siempre. Si cambia de domicilio, escriba cartas, llame por teléfono, sugiera a sus amigos y familiares que le visiten y pasen unos días con usted en su casa.

3. Aproveche todas las ocasiones que surjan para estimular una buena comunicación. Participe en excursiones, en proyectos de trabajo o voluntariado comunitario; comprométase con organizaciones internacionales solidarias que le obliguen a participar y a comunicarse; haga grupos de amigos y tertulias; intercambie libros y vea películas de interés con conocidos y coméntelas.

4. Recuerde que ayudar a otros suele ser una buena forma de comunicarse. Supere el miedo a molestar o la cobardía de acercarse a alguien e iniciar un diálogo interesante.

5. Esfuércese por comunicarse con personas de todas las edades.

6. Cuando mantenga una charla, no lance largos discursos que aburran e impidan hablar a los demás. No hable siempre del mismo tema. Procure descubrir los intereses de las personas que le rodean y facilite su discusión. Evite temas que sabe que molestan o pueden producir situaciones violentas. Muestre interés por lo que le cuentan y pregunte. Aprenda a dialogar reconociendo lo positivo de lo que le comentan y no convierta su conversación en un continuo llevar la contraria. Cuando dialogamos 
debiéramos preguntarnos si nos interesamos por las experiencias vitales de nuestro interlocutor o si sólo nos limitamos a contar nuestros problemas sin atender realmente a los otros.

\subsubsection{Preguntas para animar y centrar el debate}

1. ¿Qué diferencias existen entre el mero acto de hablar o charlar, para pasar el rato, y tener una conversación comunicativa y enriquecedora?

2. ¿Crees que comunicarnos dialogando nos ayuda a vivir con más satisfacción?

3. ¿Qué tenemos que hacer o cómo debemos dialogar para poder comunicarnos con respeto y pasando un rato agradable?

4. ¿Es cierto que es más fácil comunicarse cuando hablas de tus propias experiencias?

5. ¿Crees que es saludable contar tus problemas y dificultades a alguien en quien confíes?

6. ¿Es cierto que a muchos ancianos les resulta difícil encontrar a alguien con quien hablar?

7. ¿Se puede aprender y mejorar el nivel de vida de los mayores organizando diálogos interesantes y agradables?

8. ¿Qué sucede en nuestra sociedad que, siendo el diálogo una actividad tan importante para el ser humano y tan accesible a todos, encontramos tantas dificultades para hablar y comunicarnos satisfactoriamente?

9. ¿Crees que las prisas del mundo actual y la televisión son barreras reales para comunicarnos con la familia?

10. ¿Qué podemos hacer para facilitar la comunicación y el diálogo?

\subsection{TEMA. LOS JUEGOS DE CARTAS COMO ESPACIO DE ENCUENTRO Y DESARROLLO ENTRE LOS MAYORES}

\subsubsection{Objetivos específicos:}

1. Informar sobre el interés recreativo y las características generales de los juegos de cartas en el ocio de los mayores.

2. Promover estrategias cognitivas y hábitos de conducta que permitan reconocer y utilizar los juegos de cartas como medios de desarrollo personal.

3. Ofrecer estímulos y refuerzos positivos que afiancen un uso adecuado de los juegos de cartas, que animen a los mayores a permanecer activos e integrados socialmente.

4. Favorecer el reconocimiento, la dignidad y los aspectos positivos de los mayores valorando sus aportaciones al debate sobre el valor recreativo de los juegos de cartas.

5. Estimular la creatividad, el surgimiento de proyectos e ilusiones en torno a los juegos de cartas. 


\subsubsection{Texto (para leer, analizar y debatir):}

Existe el error, más o menos generalizado, de creer que el adulto pierde su tiempo cuando juega, pensar que el juego representa una actividad innecesaria e insustancial para el desarrollo de sus capacidades, pero lo cierto es que el juego satisface una serie de deseos y necesidades vitales en los ámbitos biológico-funcional y psicosocial.

Durante el envejecimiento el juego está relacionado con aspectos tan elementales y cotidianos como la evasión, el disfrute y la satisfacción, la actividad, el riesgo, la integración, la comunicación, la creatividad, el aprendizaje y la expresión. Desempeña funciones similares a las de cualquier otra etapa de la vida, pero adquiere las connotaciones propias que imponen las peculiaridades de la edad de los jugadores.

Si los buenos juegos son los que satisfacen las necesidades recreativas y estimulan el desarrollo pleno de los jugadores, los mejores para las personas mayores son los que, por sus cualidades, resultan más accesibles, entretienen, divierten y estimulan o mantienen el máximo de facultades que corren peligro de debilitarse durante la vejez. Entre este tipo de juegos se encuentran los juegos de cartas.

Los naipes comparten el tiempo de ocio de los ancianos con otros juegos de mesa, como el dominó, el parchís, las damas o el ajedrez; con juegos de lanzamiento, como la rana, la petanca, los bolos, y con otro tipo de juegos, como el billar. Pero, entre todas estas actividades, la más solicitada son las cartas.

Una de las razones que explica este hecho se encuentra en el dato de que los juegos de cartas funcionan como agentes de socialización. A través de este tipo de actividades, quienes acceden a los centros de reunión de jubilados asimilan y reproducen la estructura social ya vigente. Jugando los mayores que vienen de fuera se hacen un lugar entre los habituales de los hogares o clubes, y estos últimos, echando la partida, consolidan sus relaciones de amistad y acogimiento.

Otro factor que puede influir en el éxito de los naipes está conectado con la propia estructura del juego, con sus características, equiparables a las de los juegos más completos; se trata de una actividad recreativa accesible, sencilla y con una riqueza inmensa de variantes. Los juegos de cartas son accesibles, están al alcance de cualquier mayor. Para jugar, aparte de los jugadores, sólo se necesita una baraja de cartas, un recurso sencillo y simple, fácil de transportar y manejar, adecuado a las demandas físicas del organismo debilitado de los ancianos, y posee la virtud de los mejores juegos, la de ser un juego de juegos, esto es, representa toda una familia de juegos llena de creatividad y versatilidad - ¿cuántos juegos de cartas conocemos y cuántos nos quedan por conocer?- 
Por otra parte, son un tipo de actividad lúdica que facilita la asimilación de los cambios y pérdidas que se producen en la vejez, desempeña una función adaptativa en el proceso evolutivo hacia la ancianidad. En este sentido, los naipes ofrecen un espacio de evasión y catarsis que permite a los mayores liberarse de las presiones y conflictos que se les presentan; es una forma de disfrutar, de obtener satisfacciones pasando momentos agradables, con desenfado y buen humor; jugando vamos reconociendo nuestra progresiva lentitud, despistes y equivocaciones con mayor grado de tolerancia a la frustración y aceptación de nuestro deterioro personal que fuera del juego.

Los juegos de cartas también contribuyen a afianzar nuestra autoestima y autoconcepto, no sólo porque nos hacen sentirnos personas aceptadas, útiles y necesitadas dentro del grupo, sino también porque, pese a haber sufrido pérdidas en ámbitos tan importantes como el laboral, familiar, económico o funcional, comprobamos que aún conservamos control sobre el entorno que nos otorga nuestra propia habilidad jugando. Como en los sueños, en el juego se recrea la realidad, e incluso se subordina a los intereses del sujeto, los juegos de cartas aportan a los mayores el desafío, la aventura y riesgo que ya resultan inaccesibles en otros ámbitos de la vida. Son, además, una forma de frenar la mortecina pasividad que, en algunos casos, se apodera de nosotros al envejecer; atender a una partida de forma cotidiana nos exige desplazarnos, movernos ejercitando los músculos, provoca dinamismo y, por tanto, vida. Los naipes pueden utilizarse, también, como apoyo terapéutico que anime, distraiga o alivie el sufrimiento, la angustia e inactividad de las hospitalizaciones de las personas mayores; o como un ejercicio dirigido a estimular capacidades mentales básicas, como la memoria, la atención o el cálculo.

Pero, sobre todo, los juegos de cartas son una forma privilegiada de comunicación que permite a los mayores reflexionar en alto, expresarse, compartir, reconocer y aceptar con los compañeros de juego el proceso evolutivo peculiar de la vejez.

Que el mus "es algo más" que otros muchos juegos de cartas es una intuición que sostienen casi todos sus aficionados. Su éxito entre los mayores -y los no tan mayores- puede estar relacionado con el hecho de que es una actividad que iguala a todos los jugadores. Al final, la suerte y la habilidad son las únicas que deciden. Además, es un juego optimista y positivo; cuando se juega se desafía al pesimismo, siempre se espera ganar, reafirma la iniciativa del esfuerzo por optimizar y, sobre todo, el mus es un juego popular entre los mayores porque les ofrece lo que necesitan: comunicarse, divertirse y estar activos, o, dicho con las palabras de Severino Arranz, «el mus es un refugion que ofrece a los ancianos lo que en otros ámbitos no pueden conseguir.

Una partida de mus también puede ser un espacio que facilita las relaciones intergeneracionales, en el que los abuelos se comuniquen con sus nietos. 
Es un juego holista, que tiene mucho más que ofrecer a las relaciones familiares y al desarrollo personal que los nuevos esquemas de juego representados por las videoconsolas y, en general, por los videojuegos.

La respuesta a cómo un juego con cartas en el que, aparentemente, se vence sólo con cuatro jugadas: la grande, la chica, los pares y el juego, puede presentarse como un juego integral para los mayores, se debe buscar en que lo importante del juego está en las circunstancias que le rodean. Al mus no se juega sólo con los naipes, se pone en juego toda la personalidad de los participantes, se juega con los gestos, la mirada, el tono de voz, etc. Se trata de una actividad recreativa que claramente promueve una relación afectiva y social de amistad e integración, que en el ámbito psicomotriz obliga a los sujetos no sólo a coordinar movimientos manuales, sino también a ejercitar representaciones mentales sobre las jugadas, nuestras cartas, las del compañero y los adversarios.

Estimula el desarrollo cognitivo de los mayores, que juegan en tanto que se ven obligados a manejar diversos tipos de información y a resolver y comprender los cambios y dificultades que surgen en la evolución de las circunstancias. Puede utilizarse como un ejercicio de gimnasia para la mente, reconociendo e incidiendo en la posibilidad de afianzar en los jugadores cuatro características que afianzan un buen entrenamiento mental, tan necesario cuando envejecemos. Una de estas características es la fuerza mental, que se favorece en el juego de mus con el esfuerzo continuo de concentración, análisis y decisión que exige; también se impulsa la flexibilidad mental, capacidad que ejercitamos tratando de ser creativos, de utilizar jugadas que no nos delaten, que nos permitan sorprender y afianzar nuestra innovación ante los oponentes. La tercera cualidad mental que se pone en práctica jugando es la resistencia mental, cuando, porque así lo reclama la partida, mantenemos un nivel de esfuerzo superior al habitual, sin distraernos o desmoralizarnos cuando sostenemos el tipo y no renunciamos a conseguir los objetivos que nos habíamos propuesto al comenzar a jugar. Y, por último, el mus estimula la coordinación mental de los mayores empujándonos a actuar con el equilibrio y agilidad necesarios para estar a la altura de la partida; los jugadores afianzan esta aptitud mental organizando su pensamiento, buscando la virtud que se sitúa entre las capacidades del jugador y su resolución ante la jugada.

Eso sí, no puede perderse de vista, por muchos guiños que se haga al jugar, que el mus es un juego, todo un juego y nada más que un juego. Y, como tal, tiene su momento y su lugar.

\subsubsection{Preguntas para animar y centrar el debate}

1. ¿Por qué crees que los juegos de cartas son una actividad tan extendida en los centros de recreo de personas mayores? 
2. ¿Cuántos juegos de cartas conoces?

3. ¿Qué juegos de cartas son los preferidos de los mayores? ¿los mayores de otros tiempos jugaban a los mismos juegos de hoy?

4. ¿Cuál es el juego de cartas que más te gusta? ¿por qué?

5. ¿Piensas que jugar a las cartas es una buena forma de relacionarse con los nietos, para hablar con ellos y pasar un rato agradable?

6. Jugar a las cartas es bueno, pero jugar sólo a las cartas, sin interesarse por otras actividades, es empobrecedor. ¿Cómo animarías a los mayores que sólo juegan a las cartas para que realicen otras actividades, como tertulias, cinefórum, etc.?

7. ¿Piensas que apostar algo de dinero en los juegos es adecuado o incorrecto?

8. Hay muchas personas que viven solas; $¿$ crees que comprometerse a jugar un día en casa de cada uno de los jugadores o jugadoras puede ser una buena idea para estrechar amistades y mantenerse ocupado y entretenido?

9. ¿Existe algún torneo de juegos de cartas en tu barrio o localidad?

10. ¿Qué reglas de respeto y amistad deben seguirse en los juegos de cartas para que funcionen como una actividad recreativa de interés?

\section{BIBLIOGRAFÍA}

ARRANZ, S. (1996): El mus es algo más. Madrid: Ed. CDN.

COLOM, A. et alt. (1987): Modelos de intervención socioeducativa. Madrid: Narcea.

COLOM, A. y ORTE, C. (coordinadores) (2001): Gerontología educativa $y$ social. Pedagogía social y personas mayores. Palma de Mallorca: Universitat de les Illes Balears.

DELORS, J. et alt. (1996): La educación encierra un tesoro. Madrid: Santillana

ESCOLANO, A. (1995): "La educación ante los escenarios de fin de siglo", en Revista Complutense de Educación, vol.6, núm. 2, pp.169-172.

FAURE, E. et alt. (1973): Aprender a ser. Madrid: Alianza.

FERNÁNDEZ SOLIS, J. D. (1995): Dossier: Ciudad Educadora. Servicios Sociales Ayuntamiento de Alcobendas.
GARCIA GARRIDO, J. L. (coord.) (2000): La sociedad educadora. Madrid: Fundación Independiente.

GARCÍA MARTÍNEZ, A. et alt. (2000): Educación para la salud. La apuesta por la calidad de vida. Madrid: Arán.

GÚRPIDE, S. y SERRA, P. (1997): $E l$ anciano en su casa. Madrid: Consejería de Sanidad y Servicios Sociales de la Comunidad de Madr $\cdot 1$.

GÚRPIDE, S. y MADOZ, V. (1997): Una vejez joven. Cuidados de salud para personas con.... unos cuantos años. Madrid: Consejería de Sanidad y Servicios Sociales de la Comunidad de Madrid.

INSERSO (1993): Plan Gerontológico. Madrid: Instituto Nacional de Servicios Sociales

INSERSO (1993): Guía práctica para mayores. Madrid: Ministerio de Asuntos Sociales. 
INSERSO (1995): Guia directorio de Centros para personas mayores. Vol 1. Residencias. Vol 2 Hogares y Clubes. Madrid: INSERSO.

INSERSO (1996): Centros de día para personas mayores dependientes. Guia práctica. Madrid: INSERSO.

INSERSO (1996): Teleasistencia domiciliaria. Evaluación del programa. Madrid: INSERSO.

LABRADOR HERRÁIZ, C. (1998): "La educación a las puertas del siglo XXI», en Revista de Ciencias Sociales SOCIEDAD Y UTOPIA, vol. 11, pp. 209-222.

LIMÓN MENDIZÁBAL, R. (1995): «Educación para la salud en la tercera edad", en R. PEREA et alt. Educación para la salud en atención primaria. Curso de educación para la salud. Castellón/Vila-real: UNED, pp. 97-118.

LIMÓN MENDIZÁBAL, R. (1997): «Factores determinantes de la salud", en R. PEREA QUESADA, Fundamentos y justificación de la Educación para la salud. Madrid: UNED-Fundación Universidad Empresa, pp. 45-96.

LIMÓN MENDIZÁBAL, R. (1997): "La educación de las personas mayores", en A. PETRUS (Coord). Pedagogía Social. Barcelona: Ariel Educación, capítulo 13.

LIMÓN MENDIZÁBAL, R. (1999): “Educación permanente y evaluación de programas en educación social", en M. ${ }^{a}$ T. MARTIN Y M. ${ }^{a}$ L. SARRATE (coords). Evaluación y ámbitos emergentes en Animación socio-cultural. Madrid: Sanz y Torres, pp.207-234.

LIMÓN MENDIZÁBAL, R. (2001): «Datos para una Pedagogía Gerontológica", en Revista de Educación núm. 324, pp. 341-361.

LIMÓN MENDIZABAL, R. y CRESPO CABORNERO, J. A. (2001): Grupos de debate para mayores. Guía práctica para animadores. Madrid: Narcea.

ORDUÑA, G. y NAVAL, C. (editoras) (2001): Gerontología educativa. Barcelona: Ariel Educación.
ORTEGA ESTEBAN, J. (1990): "La idea de ciudad educadora a través de la historia», en J. TRILLA (coord). La ciudad educadora. Barcelona: Ajuntament Barcelona, pp. 92-102.

ORTEGA ESTEBAN, J. (1998): «Educación a lo largo de la vida o el espacio de la educación social», en L. PANTOJA (coord). Nuevos espacios de la educación social Bilbao: Mensajero, pp 161-182.

ORMAZA, I. (1995): Grupos de apoyo en la vejez. Bilbao: Gobierno Vasco.

PEREA QUESADA, R (1994). Educación para la salud pública. Curso de Educación para la salud. Madrid: UNED.

PETRUS ROTGER, A. (2001): "Nuevas experiencias de intervención socioeducativa hacia las personas mayores», en A. COLOM y C. ORTE (Coords). Gerontología educativa $y$ social,. Palma: Universitat de les Illes Balears, pp 611-647.

REVISTA ESPAÑOLA DE GERIATRÍA Y GERONTOLOGÍA (1994): Monográfico: Salud y asistencia sanitaria, núm. 29. Madrid

REVISTA CIUDAD ESCOLAR Y UNIVERSITARIA (2001): Monográfico: Pozuelo, ciudad educadora, núm. 39. Madrid: Ayuntamiento de Pozuelo de Alarcón.

RODRIGUEZ, P.; SANCHO M.' T., et alt. (1995): Residencias para personas mayores. Manual de orientación. Barcelona: SG Editores.

RODRÍGUEZ, P.; SANCHO, M. ${ }^{\mathrm{a}} \mathrm{T}$. et alt. (1997): El servicio de Ayuda a domicilio. Programación del servicio. Formación para auxiliares. Madrid: Panamericana.

SANCHO, M. T. (1994): «Un lugar para vivir. Sistemas alternativos de alojamiento", en Revista de la Sociedad Española de Geriatría y Gerontología, núm. 29.

SARRAMONA, J. (1986): Prólogo a la obra de J. PAZ. Instituciones educativas para adultos en España. Madrid: Seco Olea. 
SARRAMONA, J.; VÁZQUEZ, G. y COLOM, A. (1998): Educación no formal. Barcelona: Ariel Educación.

TAMAYO, J. (1995). "La cultura como forma de vida y comunicación", en Jornadas por una vejez activa, Barcelona: F.C.M. pp. 227-237.
TRILLA BERNET, J. (1993). Otras educaciones. Animación sociocultural, formación de adultos y ciudad educativa. Barcelona: Anthropos.

VELÁZQUEZ CLAVIJO, M. et al (1999): Guia de Programas Universitarios de Personas Mayores. Madrid: Ministerio de Trabajo y Asuntos Sociales.

\section{RESUMEN}

- Mantenerse sano y con el mayor grado de autonomía y bienestar es uno de los requisitos más importantes para disfrutar de una jubilación satisfactoria

- Cada persona tiene la potencialidad para asumir la responsabilidad que le corresponde en el cuidado de su propia salud.

- En la actualidad hay una mayor demanda de información sobre el cuidado de la salud. Esto se debe a que hay una mayor conciencia de que la salud es, en gran medida, responsabilidad nuestra y no de los profesionales de la salud (médicos, sanitarios...), como antes se creía.

- En la implantación y desarrollo de las disciplinas "Educación para la salud", "Educación de las personas mayores" o "Gerontología educativa" en la Universidad española, ha sido fundamental la progresiva consolidación de la Pedagogía Social.

- Desde la perspectiva de la Ciudad educadora, analizamos en este artículo los distintos Espacios para Mayores donde se desarrollan programas de educación para la salud a través de talleres (de memoria, de autoestima, de pensamiento positivo y alegría de vivir, de hábitos saludables, de técnicas de relajación...), seminarios, grupos de debate, conferencias, cursos, charlas...

- Estos espacios han pasado de tener un carácter asistencial en los años setenta, a una concepción socio-educativa y cultural, dirigida a favorecer la participación y formación continua de los adultos mayores, en la entrada al nuevo milenio.

- Son espacios de aprendizaje, en definitiva, espacios de vida donde compartir información, experiencias y formación con el fin de envejecer de manera saludable y potenciar el desarrollo personal en esta nueva etapa de la vida. 
- Por último, presentamos un curso sobre «Promoción de la salud y bienestar de los mayores" para ser desarrollado a través de grupos de debate.

Palabras clave: Educación permanente, ciudad educadora, educación para la salud, espacios para mayores, autocuidado, salud y bienestar, grupos de debate.

\section{ABSTRACT}

- To stay healthy and with the highest degree of autonomy and well-being is one of the most important requirements to enjoy a satisfactory retirement.

- Each person has the potentiality to assume the corresponding responsibility in the care of his/her own health.

- At the present time there is a bigger demand for information about health care. This is due to the increasing awareness on health being, greatly, our responsibility and not only the professionals' of health (doctors, sanitariums...), as it was the traditional.

- In the implementation and development of the disciplines "Education for health", "Elder Education" or "Educational Gerontology» in the Spanish University, it has been fundamental the progressive consolidation of Social Pedagogy.

- Assuming the approach of the educational City, we analyse in this article, the different Spaces for The Elder where education educational health programs are being developed through workshops (for memory, self-esteem, positive thought and happiness of living, healthy habits, techniques for relaxation...), seminars, groups, conferences, courses, chats...

- These spaces have changed from having an assistance character in the seventies, to a partner-educational and cultural conception, directed to favour participation and adults' continuous training, at the beggining of the new millennium.

- They are learning spaces, in short, spaces of life to share information, experiences and education with the purpose of aging in a healthy way and to promote personal development in this new stage of life.

- Finally, we present a course on "Promotion of health and well-being of the Elder" to be developed through debate groups.

Key Words: permanent education, educational city, education for health, spaces for old people, self-care, health and well-being, debate groups. 